\section{Estudo da mortalidade dos médicos no Estado de São Paulo, Brasil, no período 2000-2009}

\author{
Physicians' mortality in São Paulo State, Brazil, \\ 2000-2009
}

\author{
Estudio de la mortalidad de los médicos en el \\ estado de São Paulo, Brasil, durante el período \\ de 2000 a 2009
}

1 Departamento de Medicina
Preventiva, Universidade
Federal de São Paulo,
São Paulo, Brasil.
2 Departamento de
Psiquiatria, Universidade
Federal de São Paulo,
São Paulo, Brasil
Correspondência
Z. M. Sanchez
Centro Brasileiro de
Informações sobre Drogas e
Psicotrópicos, Departamento
de Medicina Preventiva,
Universidade Federal de
São Paulo.
Rua Borges Lagoa 1341,
São Paulo, SP 04038-034,
Brasil.
zila.sanchez@gmail.com

Abstract

This study presents the distribution of causes of death among physicians in the State of São Paulo, Brazil, from 2000 to 2009. The study used the database of the Mortality Information System, Secretariat of Health Surveillance, Brazilian Ministry of Health, limited to death certificates in which the item on occupation was reported as "physician". Underlying causes of death were classified according to ICD-10 chapters. The study showed that cardiovascular diseases were the leading cause of death among physicians, followed by cancer and respiratory diseases. Under age 40, doctors of both genders died mainly from external causes. Among cancer deaths, breast cancer was the leading cause in women and lung cancer the first cause in men. The results show the need to promote adequate health habits and disease prevention among physicians, seeking to raise awareness in this group of professionals.

Physicians; Mortality; Cardiovascular Diseases; Neoplasms
Zila M. Sanchez 1

Hamer N. Palhares Alves 2

Luiz Antônio Nogueira-Martins 2

Mariangela C. Oliveira Prado 1

\section{Resumo}

O presente estudo apresenta a distribuição das causas de morte de médicos do Estado de São Paulo, Brasil, cujos óbitos ocorreram entre os anos de 2000 e 2009. Utilizou-se o banco de dados do Sistema de Informações sobre Mortalidade, do Ministério da Saúde, limitando-se às declarações de óbito cujo campo de ocupação estava preenchido como "médico". As causas básicas de morte foram classificadas de acordo com os capitulos da CID-10. O estudo revela que doenças do aparelho circulatório foram a principal causa de morte entre os médicos na última década, seguidas das neoplasias e doenças do aparelho respiratório. As causas externas constituíram as principais causas de morte abaixo de 40 anos. Câncer da mama foi responsável pela maior parte dos óbitos por neoplasias entre mulheres, enquanto entre os homens a neoplasia de pulmão ocupou a primeira posição. Sugere-se a necessidade de se estimular hábitos adequados de saúde e prevenção de doenças entre os médicos, buscando informar e sensibilizar este grupo de profissionais.

Médicos; Mortalidade; Doenças

Cardiovasculares; Neoplasias 


\section{Introdução}

A análise das causas de morte é fundamental para o monitoramento da situação de saúde e planejamento de intervenções visando a reduzir a mortalidade por causas evitáveis 1 . No Brasil, o Sistema de Informações sobre Mortalidade (SIM) do Ministério da Saúde reúne informações sobre a ocorrência e característica dos óbitos registrados nos municípios, estados e regiões brasileiras.

No caso dos médicos há evidências nacionais $2,3 \mathrm{e}$ internacionais ${ }^{1}$ de que, apesar do conhecimento acadêmico na área de cuidados à saúde, a mortalidade deste grupo pode ocorrer em faixas etárias inferiores às da população de mesmo nível socioeconômico. No entanto, isso ainda não é consenso, visto que médicos podem apresentar idade média de morte maior que a de dentistas e engenheiros 4 , sugerindo que alguns aspectos do estilo de vida destes profissionais estejam influenciando no perfil de mortalidade 2 .

Pela escassez de dados nacionais sobre o assunto, objetiva-se, neste trabalho, conhecer a distribuição das causas de morte de médicos do Estado de São Paulo, Brasil, falecidos entre 2000 a 2009.

\section{Método}

Realizou-se estudo de série histórica com dados secundários provenientes do SIM, referentes ao período de 2000 a 2009. Estudaram-se os óbitos de residentes no Estado de São Paulo cujo campo "ocupação" fosse preenchido como "061" (médico). Excluíram-se as declarações com esse campo em branco (16\% do total). Foram calculadas dentro de cada categoria de sexo dos falecidos: (1) as médias de idade dos óbitos; (2) a contribuição percentual de faixas etárias selecionadas no total dos óbitos; (3) a mortalidade proporcional por capítulo da CID-10 (Classificação Internacional de Doenças, 10a revisão), destacando-se aqueles que, no seu conjunto, concentraram mais de $90 \%$ dos óbitos e; (4) a contribuição percentual das principais causas de morte no total de óbitos por neoplasias e causas externas.

O banco de dados foi codificado no Microsoft Access (Microsoft Corp., Estados Unidos) e analisado em Stata 11 (Stata Corp., College Station, Estados Unidos). Não foram realizados testes de hipóteses por não se tratar de uma amostra, visto que todos os casos de óbitos em médicos do Estado de São Paulo foram analisados.

\section{Resultados}

Foram obtidos dados de 2.927 declarações de óbitos de médicos residentes no Estado de São Paulo, falecidos entre os anos de 2000 a 2009.

No total das declarações de óbito analisadas houve predominância de: sexo masculino $(86,2 \%)$, brancos $(94 \%)$, casados $(65 \%)$ e residentes do Município de São Paulo (54\%). A idade em que ocorreu o óbito variou de 23 a 104 anos.

Em nenhuma das declarações de óbito de médicos foram encontradas causas básicas sem preenchimento (missing), e apenas 44 delas $(1,5 \%)$ estavam classificadas como causas mal definidas (R99).

No período, foram registrados 387 óbitos de médicas e 2.540 de médicos. A análise da idade de morte evidenciou que mulheres médicas morreram, em média, dez anos mais jovens do que os homens médicos. A idade média de morte foi de 59,2 anos para mulheres e de 69,1 para os homens. Nota-se diferença na idade média de morte entre os gêneros.

Ao longo da década (2000 a 2009), tanto entre homens quanto entre mulheres houve aumento da idade média de morte.

A Figura 1 apresenta curvas de mortalidade proporcional por faixa etária. Enquanto a concentração de óbitos femininos deu-se entre 5059 anos, a dos óbitos masculinos ocorreu entre 80-89 anos de idade.

A Tabela 1 mostra que as doenças do aparelho circulatório foram as principais causas de morte entre os médicos $(29,7 \%)$, seguidas pelas neoplasias $(27,9 \%)$, doenças do aparelho respiratório $(10,7 \%)$ e causas externas $(8,9 \%)$. Em números absolutos, as causas externas geraram de 21 a 34 mortes por ano na última década, enquanto as doenças do aparelho circulatório fatais acometeram em média 87 médicos a cada ano.

A mortalidade proporcional, quando analisada por sexo, mostrou diferenças nas duas primeiras causas de morte. Para as médicas, as neoplasias superaram as doenças do aparelho circulatório e se tornaram a causa básica principal; para os médicos, as principais causas foram as doenças do aparelho circulatório (Tabela 1). Já em estratos etários específicos as primeiras causas de morte diferiram entre homens e mulheres apenas na faixa etária de 40-49 anos e de $\geq 80$ anos (Tabela 2).

Entre os homens, o câncer de pulmão (com $18 \%$ dos óbitos) foi a neoplasia que mais contribuiu para os óbitos, seguida pelo câncer da próstata $(12 \%)$ e de cólon e reto (11\%). Entre as médicas, o câncer da mama foi responsável por $30 \%$ dos óbitos em que as neoplasias foram a causa 
Figura 1

Mortalidade proporcional por idade de acordo com o sexo, entre médicos residentes no Estado de São Paulo, Brasil, falecidos entre 2000 e 2009.

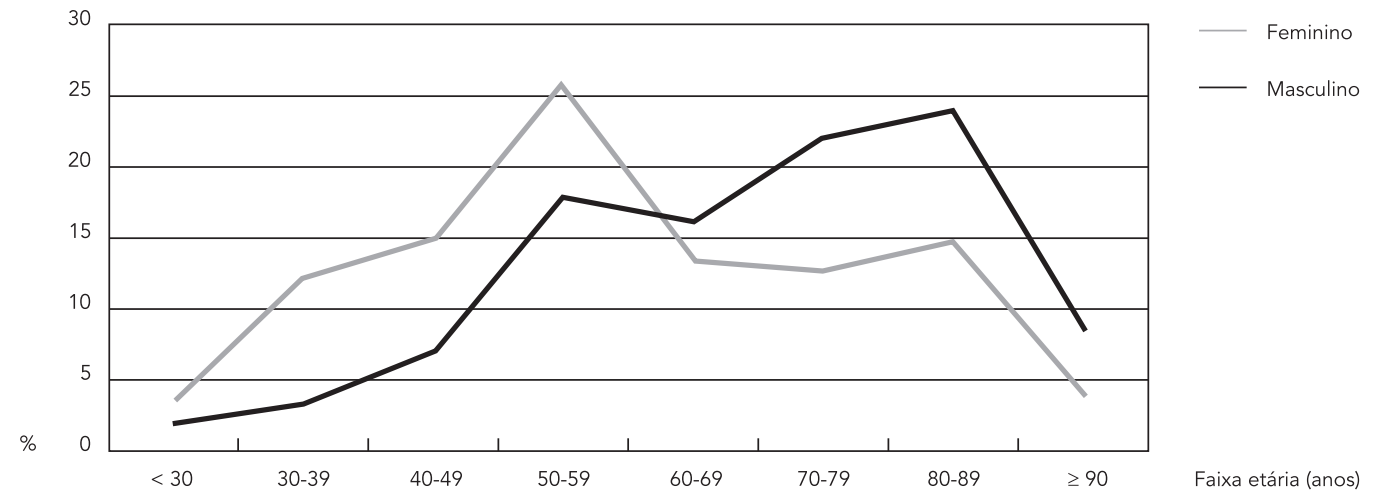

Fonte: Departamento de Informática do SUS. Sistema de Informações sobre Mortalidade (http://tabnet.datasus.gov.br/ tabdata/sim/dados/indice.htm, acessado em Nov/2011).

Tabela 1

Mortalidade proporcional de médicos por capítulo da Classificação Internacional de Doenças, 10ạ revisão (CID-10). Estado de São Paulo, Brasil, $2000-2009$.

\begin{tabular}{|c|c|c|c|c|c|c|}
\hline \multirow[t]{2}{*}{ Capítulo da CID-10 } & \multicolumn{2}{|c|}{ Masculino } & \multicolumn{2}{|c|}{ Feminino } & \multicolumn{2}{|c|}{ Total } \\
\hline & $\mathbf{n}$ & $\%$ & $\mathbf{n}$ & $\%$ & $\mathbf{n}$ & $\%$ \\
\hline I. Algumas doenças infecciosas e parasitárias & 69 & 2,7 & 14 & 3,6 & 83 & 2,8 \\
\hline II. Neoplasias (tumores) & 683 & 26,9 & 135 & 34,9 & 818 & 27,9 \\
\hline III. Doenças do sangue e dos órgãos hematopoéticos e alguns transtornos imunitário & 10 & 0,4 & 3 & 0,8 & 13 & 0,4 \\
\hline IV. Doenças endócrinas nutricionais e metabólicas & 124 & 4,9 & 12 & 3,1 & 136 & 4,6 \\
\hline V. Transtornos mentais e comportamentais & 25 & 1,0 & 3 & 0,8 & 28 & 1,0 \\
\hline VI. Doenças do sistema nervoso & 93 & 3,7 & 8 & 2,1 & 101 & 3,5 \\
\hline IX. Doenças do aparelho circulatório & 797 & 31,4 & 73 & 18,9 & 870 & 29,7 \\
\hline X. Doenças do aparelho respiratório & 273 & 10,7 & 40 & 10,3 & 313 & 10,7 \\
\hline XI. Doenças do aparelho digestivo & 129 & 5,1 & 18 & 4,7 & 147 & 5,0 \\
\hline XIV. Doenças do aparelho geniturinário & 59 & 2,3 & 8 & 2,1 & 67 & 2,3 \\
\hline XX. Causas externas & 207 & 8,1 & 53 & 13,7 & 260 & 8,9 \\
\hline Todos os demais & 71 & 2,8 & 20 & 5,2 & 91 & 3,1 \\
\hline Total & 2.540 & 100,0 & 387 & 100 & 2.927 & 100,0 \\
\hline
\end{tabular}

Fonte: Departamento de Informática do SUS. Sistema de Informações sobre Mortalidade (http://tabnet.datasus.gov.br/tabdata/sim/dados/indice.htm, acessado em Nov/2011).

básica, seguido pelo câncer de cólon e reto (10\%) e pulmão (9\%).

Acidentes automobilísticos (incluindo os atropelamentos) contribuíram com quase $40 \%$ das mortes por causas externas em ambos os sexos. Os suicídios representaram a segunda cau- sa de morte dos profissionais médicos entre as causas externas e foram mais frequentes entre as mulheres. 
Tabela 2

Mortalidade proporcional por grandes grupos de causas, de acordo com o sexo e a faixa etária, entre médicos residentes no Estado de São Paulo, Brasil, falecidos entre 2000 e 2009.

\begin{tabular}{|c|c|c|c|c|}
\hline \multirow[t]{2}{*}{ Faixa etária (anos) } & \multicolumn{2}{|c|}{ Masculino } & \multicolumn{2}{|c|}{ Feminino } \\
\hline & $\begin{array}{c}\text { Principal causa } \\
\text { básica }\end{array}$ & $\begin{array}{c}\% \text { de mortes nesta } \\
\text { categoria }\end{array}$ & $\begin{array}{l}\text { Principal causa } \\
\text { básica }\end{array}$ & $\begin{array}{c}\% \text { de mortes nesta } \\
\text { categoria }\end{array}$ \\
\hline$<30$ & Causas externas & 76 & Causas externas & 57 \\
\hline $30-39$ & Causas externas & 44 & Causas externas & 35 \\
\hline $40-49$ & $\begin{array}{c}\text { Doenças do aparelho } \\
\text { circulatório }\end{array}$ & 26 & Neoplasias & 50 \\
\hline $50-59$ & Neoplasias & 33 & Neoplasias & 47 \\
\hline $60-69$ & Neoplasias & 34 & Neoplasias & 43 \\
\hline 70-79 & $\begin{array}{c}\text { Doenças do aparelho } \\
\text { circulatório }\end{array}$ & 35 & $\begin{array}{c}\text { Doenças do aparelho } \\
\text { circulatório }\end{array}$ & 29 \\
\hline$\geq 80$ & $\begin{array}{c}\text { Doenças do aparelho } \\
\text { circulatório }\end{array}$ & 32 & $\begin{array}{c}\text { Doenças do aparelho } \\
\text { respiratório }\end{array}$ & 35 \\
\hline
\end{tabular}

Fonte: Departamento de Informática do SUS. Sistema de Informações sobre Mortalidade (http://tabnet.datasus.gov.br/ tabdata/sim/dados/indice.htm, acessado em Nov/2011).

\section{Discussão}

Apesar da relevância deste estudo como alerta à comunidade científica, limitações potenciais devem ser destacadas: (1) a possível perda de casos por preenchimento inadequado do campo "ocupação"; (2) a impossibilidade de estimar o risco de morrer por falta de informação sobre a distribuição da população de médicos por faixa etária. O envelhecimento na profissão entre os homens é bem maior do que entre as mulheres (que ingressaram mais recentemente neste meio) e, como o risco de morte eleva-se com o aumento da idade, surge a impossibilidade de se fazer comparações adequadas entre duas populações de estrutura etária distinta.

Por sua vez, um diferencial dos registros de óbitos dos médicos do Estado de São Paulo é a baixa ocorrência de mortes por causas mal definidas. Enquanto a proporção de óbitos por essas causas no Estado de São Paulo no período foi de $7 \%$, para os médicos ela ficou em $1,5 \%$, tornando a análise das causas de morte neste grupo bastante válida.

Entre as médicas as neoplasias superaram as doenças cardiovasculares e constituíram a principal causa de morte nas idades entre 30 e 59 anos. Com referência às principais causas de óbito entre as neoplasias, a distribuição foi diferente da observada na população geral da mesma faixa etária 5,6. A boa notícia para as médicas foi a pequena participação percentual do câncer do colo do útero $(4 \%)$, patologia com altos potenciais de prevenção e cura quando diagnosticado e tratado precocemente 7 . Por outro lado, o câncer da mama é a neoplasia que mais mata médicas e mulheres da população em geral.

Com relação às causas externas, diferentemente do que ocorre na população em geral ${ }^{8}$, a participação deste grupo foi proporcionalmente mais importante para mulheres do que para homens, incluindo acidentes automobilísticos e suicídio. O problema com a mortalidade proporcional é que a soma de todas as parcelas tem de totalizar $100 \%$, não sendo possível dizer se o crescimento de uma se deve à redução de outra 9. No entanto, é importante destacar que estudos evidenciam que médicos de ambos os sexos estão claramente hiper-representados entre as vítimas de suicídio 10 .

Chama também a atenção o fato de a média de idade de morte das mulheres médicas ser bem inferior à dos homens. Sabe-se que, no Brasil, o sexo masculino apresenta taxa de mortalidade mais elevada e menor esperança de vida ao nascer (77 anos para as mulheres e 69 anos para os homens em 2009) 11. É preciso lembrar, no entanto, a diferença entre os sexos na distribuição etária dos médicos, refletindo a entrada recente das mulheres na profissão 12 .

Mesmo levadas em conta essas limitações, um dos resultados obtido neste estudo é digno de nota: a elevada participação relativa das causas externas no obituário feminino. A mortalidade proporcional não é um indicador de risco, mas permite identificar áreas prioritárias para ação. 
Os resultados aqui demonstrados nos permitem supor que as médicas do Estado de São Paulo, no período de 2000 a 2009, se ressentiram de uma prática profissional geradora de estresse. A questão envolve a natureza desgastante da profissão, condições de trabalho precárias e baixa remuneração, a necessidade constante de atualização, o aumento da competitividade decorrente do crescente número de profissionais formados a cada ano e, o aumento de processos na esfera judicial 13 .

Resta saber se os profissionais que cuidam dos outros estão descuidando de si, questão que não pode ser respondida por dados secundários de mortalidade, como os deste trabalho.

\section{Resumen}

El estudio presenta la distribución de las causas de muerte de los médicos en el estado de São Paulo, Brasil, entre los años 2000 y 2009. Se utilizó la base de datos del Sistema de Información sobre Mortalidad del Ministerio de Salud, limitándose a los certificados de defunción, cuya ocupación del finado figuraba como "médico". Las principales causas de muerte fueron clasificadas de acuerdo con los capítulos del CIE-10. El estudio muestra que las enfermedades cardiovasculares son la principal causa de muerte entre los médicos durante la última década, seguido del cáncer y las enfermedades respiratorias. Las causas externas fueron las principales causas de muerte en menores de 40 años. El cáncer de mama es responsable de la mayoría de las muertes por cáncer entre las mujeres, mientras que entre los hombres el cáncer de pulmón ocupa el primer lugar. Se sugiere la necesidad de estimular los hábitos adecuados de salud y prevención de enfermedades entre los médicos, buscando informar y sensibilizar a este colectivo de profesionales.

Médicos; Mortalidad; Enfermedades Cardiovasculares; Neoplasias

\section{Colaboradores}

Z. M. Sanchez foi responsável pela coordenação do estudo, análises de dados e redação da primeira versão do manuscrito. H. N. P. Alves foi responsável pelo planejamento do estudo, revisão da literatura e discussão. L. A. Nogueira-Martins contribuiu com a interpretação dos resultados e revisão do texto. M. C. O. Prado contribuiu com a redação do texto e revisão crítica do mesmo.

\section{Agradecimentos}

Agradecemos ao CREMESP pelo financiamento do presente estudo. 


\section{Referências}

1. Aasland OG, Hern E, Haldorsen T, Ekeberg O. Mortality among Norwegian doctors 1960-2000. BMC Public Health 2011; 11:173.

2. Arcanjo HR, Silva AC, Miranda PSC, Pinheiro TMM. Estudo da morbi-mortalidade entre médicos da Região Metropolitana de Belo Horizonte, Minas Gerais. Considerações especiais sobre o suicídio neste grupo de trabalhadores. Rev Méd Minas Gerais 2000; 10:72-7.

3. Pompermaier JA. Mortalidade de médicos em Santa Catarina. Revista do CREMESC 2011; 114:8-11.

4. Antunes JLF, Macedo MM, Araujo ME. Análise comparativa da proporção de óbitos segundo causas, de dentistas na cidade de São Paulo. Cad Saúde Pública 2004; 20:241-8.

5. Secretaria de Vigilância em Saúde, Ministério da Saúde. Evolução da mortalidade no Brasil. http:// portal.saude.gov.br/portal/arquivos/pdf/capitu lo3_sb.pdf (acessado em 25/Out/2012).

6. Rodrigues AD, Bustamante-Teixeira MT. Mortalidade por câncer de mama e câncer de colo do útero em município de porte médio da Região Sudeste do Brasil, 1980-2006. Cad Saúde Pública $2011 ; 27: 241-8$

7. Instituto Nacional de Câncer. Estimativa 2008: incidência de câncer no Brasil. http://bvsms.saude. gov.br/bvs/publicacoes/estimativa_incidencia_ cancer 2008.pdf (acessado em 25/Out/2012).
8. Mello-Jorge MHP, Gawryszewski MP, Latorre MR Análise dos dados de mortalidade. Rev Saúde Pública 1997; 31:5-25.

9. Marcopito LF, Berlin JA. A method for calculating age-weighted death proportions for comparison purposes. Int J Epidemiol 1998; 27:1044-52.

10. Gold KJ, Sen A, Schwenk TL. Details on suicide among US physicians: data from the National Violent Death Reporting System. Gen Hosp Psychiatry 2013; 35:45-9.

11. Instituto Brasileiro de Geografia Estatística. Observações sobre a evolução da mortalidade no Brasil: o passado, o presente e perspectivas. Rio de Janeiro: Instituto Brasileiro de Geografia e Estatística; 2010.

12. Sheffer M, Biancarelli A, Cassenote A. Demografia médica no Brasil: dados gerais e descrições de desigualdades. http://www.cremesp.org.br/pdfs/ demografia_2_dezembro.pdf (acessado em 08/ Mar/2012).

13. Nogueira-Martins LA, Nogueira-Martins MCF. O exercício atual da medicina e a relação médicopaciente. Rev Bras Clín Ter 1998; 24:59-64.

Recebido em 24/Jun/2012

Versão final reapresentada em 04/Mar/2013

Aprovado em 24/Abr/2013 